\title{
Asociación entre albuminuria y mortalidad en adultos mayores con pie diabético en el servicio de clínica de día en el Centro Médico Naval (2010-2015)
}

\section{Association between albuminuria and mortality in elderly subjects with diabetic foot seen in the outpatient clinic of the Peruvian Navy Medical Center (2010-2015)}

Correspondencia Héctor Estremadoyro Santillan estremadoyro92@gmail.com

Recibido: 19/04/2020

Arbitrado por pares

Aprobado: 04/06/2020

Citar como: Estremadoyro-Santillan $H$, Runzer-Colmenares F, Parodi JF. Asociación entre albuminuria y mortalidad en adultos mayores con pie diabético en el servicio de clínica de día en el Centro Médico Naval (2010-2015). Acta Med Peru. 2020;37(2):156-62. doi: https://doi. org/10.35663/amp.2020.372.944
Héctor A. Estremadoyro-Santillan ${ }^{1, a}$, Fernando M. Runzer-Colmenares ${ }^{1,2, b}$, José F. Parodiª,b

1 Facultad de Ciencias de la Salud, Carrera de Medicina Humana. Universidad Científica del Sur. Lima, Perú.

2 Centro de Investigación del Envejecimiento, Facultad de Medicina Humana. Universidad de San Martín de Porres. Lima, Perú.

a Médico-cirujano, b médico geriatra

\section{RESUMEN}

Objetivo: determinar la relación entre albuminuria y mortalidad en adultos mayores con pie diabético atendidos en la Clínica de Día del Servicio de Geriatría del Centro Médico Naval del Perú “Cirujano Mayor Santiago Távara” en el periodo 2010-2015. Materiales y Métodos: estudio cuantitativo, observacional, analítico, cohorte retrospectiva. Se incluyeron datos de 89 pacientes adultos mayores, que cumplieron los criterios de selección. Se realizó un análisis descriptivo incluyendo las principales variables como fallecimiento durante el estudio, comorbilidades y variables sociodemográficas. Posteriormente, se hizo un análisis bivariado en base a supervivencia al final del estudio donde se analizaron todas las covariables en base a la variable principal del estudio (mortalidad). Resultados: la edad promedio fue de 79,3 $\pm 7,2$ años con predominancia del sexo masculino $(58,4 \%)$, encontramos que 52 participantes $(58,4 \%)$ tenían antecedentes de albuminuria y que el $7,9 \%$ de la población falleció durante el estudio, de los cuales el $100 \%$ presentaba albuminuria. Finalmente, al igual que en el caso de albuminuria, todos los pacientes con antecedente de amputación por pie diabético fallecieron durante el seguimiento. Conclusión: se encontró relación significativa entre la presencia de albuminuria y mortalidad en los adultos mayores con pie diabético incluidos en el estudio.

Palabras clave: Albuminuria; Pie diabético; Mortalidad; Anciano (fuente: DeCS BIREME). 


\section{ABSTRACT}

Objective: To determine the relationship between albuminuria and mortality in elderly subjects with diabetic foot seen in the outpatient clinic in Cirujano Mayor Santiago Tavara Peruvian Navy Medical Center between 2010 and 2015. Materials and Methods: This is a quantitative, observational, analytical and retrospective cohort study. Data from 89 elderly subjects who complied with selection criteria was entered. A descriptive analysis was performed in which the following variables were included: death during the study, comorbidities, and sociodemographic data. Afterwards, a bivariate analysis based on survival at the end of the study was performed, where all covariates based on the main variable of the study (mortality) were assessed. Results: Average age of subjects was $79.3 \pm 7.2$ years, and they were mainly male $(58.4 \%)$. We also found that 52 subjects $(58.4 \%$ ) had a past history of albuminuria, and that $7.9 \%$ of the studied population died during the study. All of them (100\%) had albuminuria. Finally, as it was the case with albuminuria, all subjects who had undergone amputation because of diabetic foot died during follow-up. Conclusion: A significant relationship between albuminuria and mortality was found in elderly subjects with diabetic foot who were included in this study.

Keywords: Albuminuria; Diabetic foot; Mortality; Aged (source: MeSH NLM).

\section{INTRODUCCIÓN}

La diabetes tipo 2 (DM2), enfermedad de alta prevalencia a nivel mundial, afecta aproximadamente 387 millones de personas, con un índice de mortalidad en aumento, es la octava causa de muerte en el mundo, producto de múltiples manifestaciones y complicaciones tardías ${ }^{[1,2]}$. Se calcula que dichas complicaciones reducen la expectativa de vida, en promedio, hasta once años en hombres y catorce años en mujeres, por encima de los 65 años ${ }^{[3]}$.

Una de estas complicaciones es el pie diabético, el cual implica daño en la microvasculatura arterial periférica. Su frecuencia aumenta anualmente, proporcional a un mayor tiempo de enfermedad, es así que se calcula que la prevalencia de las alteraciones podológicas es alrededor del $10 \%$, correspondiendo el 2-10\% a úlceras, y entre 0,2 a $2 \%$ a amputaciones principalmente entre la población adulta mayor ${ }^{[4]}$. Adicionalmente, se debe considerar que existen otras complicaciones que nos indican alteración de la perfusión sistémica como el daño a nivel de la barrera de filtrado glomerular, manifestado mediante la albuminuria como marcador de daño microvascular tardío, que ha sido asociado a enfermedad renal crónica y muerte ${ }^{[5]}$.

Estudios anteriores como el de Guerrero et al. (1998) ${ }^{[6]}$ ya demostraban una relación entre el desarrollo de albuminuria y presencia de úlceras de pie diabético, sugiriendo que su temprana identificación podía llevar a retrasar la evolución de ambas entidades. En otro estudio, llevado a cabo por Akha et al. (2010) ${ }^{[7]}$, se describe la correlación entre amputación del pie diabético y nefropatía diabética, siendo esta asociación de peor pronóstico en la supervivencia de estos pacientes. La misma investigación estima que las complicaciones podológicas son el doble en aquellos pacientes con fallo renal, y la prevalencia de amputación es 6,5 a 10 veces mayor.

Sin embargo, existen pocos estudios de pacientes diabéticos con manifestaciones tardías y su impacto en la mortalidad, específicamente cohortes de pacientes adultos mayores con pie diabético; con la finalidad de analizar factores de riesgo de mortalidad, los cuales podrían ser diferentes a los de la población de diabéticos en general tomando en cuenta el tiempo de enfermedad, el soporte social evidenciado con el estado civil y el control de la diabetes mediante la glicemia y hemoglobina glicosilada.

El presente estudio tiene como objetivo determinar la relación entre albuminuria y mortalidad en adultos mayores con pie diabético atendidos en la clínica de día del servicio de Geriatría del Centro Médico Naval del Perú "Cirujano Mayor Santiago Távara" en el periodo 2010-2015.

\section{MATERIALES Y MÉTODOS}

\section{Diseño y tipo de estudio}

Estudio de tipo cuantitativo, observacional, analítico, cohorte retrospectiva, con análisis secundario de una base de datos ${ }^{[8]}$.

\section{Población y muestra}

Se incluyeron pacientes adultos mayores de un estudio previo ${ }^{[8,9]}$, de ambos sexos, que tuvieron como diagnóstico pie diabético, provenientes de atención ambulatoria. Estos pacientes iniciaron seguimiento en la unidad de clínica de día destinada para la cura de heridas de pie diabético, insuficiencia vascular y úlceras por presión perteneciente al Servicio de Geriatría del Centro Médico Naval "Cirujano Mayor Santiago Távara". Este centro atiende aproximadamente 1600 pacientes ambulatorios por mes, entre personal militar en retiro y sus familiares directos.

El estudio original se llevó a cabo durante un periodo de cinco años, entre los años 2010 hasta el 2015; sin embargo, la mortalidad fue evaluada de manera trimestral o semestral hasta los seis meses posteriores al final del estudio, mediante informes de la Oficina de Epidemiología del Centro Médico Naval. El tiempo de seguimiento, en promedio, fue de 1044,5 $\pm 10,4$ días. 
El tipo de muestreo empleado fue no probabilístico, donde la muestra de nuestro estudio fue la totalidad del tamaño muestral calculado por los investigadores principales del estudio original.

\section{Cálculo de la potencia estadística}

Para el cálculo se utilizó el estudio de K. Matsushita et al. (2010) ${ }^{[10]}$ donde se expone que hay un riesgo de muerte por cualquier causa de 1,14 veces en pacientes con albuminuria en comparación a los pacientes sin albuminuria. Con esta información y mediante el Software OpenEpi (Open Source Epidemiologic Statistics for Public Health) ${ }^{[11]}$, se calculó que se necesita un tamaño muestral de 80 registros (40 casos de albuminuria y 40 controles sin albuminuria), considerando una potencia estadística del $80 \%$. Para el presente estudio se contó con datos de 89 participantes.

\section{Criterios de selección}

Se incluyó datos de participantes adultos mayores, así como de sus familiares directos, con 60 años o más, diagnóstico de pie diabético grado I o mayor (según escala Wagner), provenientes de consulta ambulatoria del Servicio de Geriatría del hospital, que accedieron formar parte del estudio original.

\section{Variables del estudio}

Se consideró como variable independiente a la presencia de albuminuria; definida en el estudio como la presencia de albúmina en orina de 24 horas con un punto de corte de 30 a 300 $\mathrm{mg} 0$ «microalbuminuria» ${ }^{[12]}$. Se consideró su presencia durante los tres meses previos al inicio del seguimiento, en la que pudo ser diagnosticada mediante dos medidas de albúmina en orina, quedando registrado el antecedente en la historia clínica por parte de los médicos nefrólogos.

La variable dependiente fue la mortalidad, definida como la expresión del fallecimiento de un individuo durante el seguimiento del estudio original, corroborado mediante información de la Oficina de Epidemiología del Centro Médico Naval del Perú.

Como covariable, se evaluó el control de la DM2; definido según las recomendaciones de la Asociación Americana de Diabetes (2020) ${ }^{[13]}$ como adecuado, si los datos de glicemia pre-prandial oscilaban entre $80-130 \mathrm{mg} / \mathrm{dL}$ y/o Hemoglobina glicosilada $(\mathrm{HbA} 1 \mathrm{c})<7 \%$, considerando los valores de los 3 meses previos al inicio del seguimiento.

Adicionalmente, se recolectó información sobre variables sociodemográficas como la edad, tiempo de enfermedad (en años), sexo, grado de instrucción (analfabeto/Incompleta, secundaria Completa, educación técnica y educación superior), estado civil (soltero (a), casado (a), viudo (a), divorciado (a) y separado (a)). Así mismo, se evaluaron variables clínicas como la presencia de amputación (sin especificar tipo de amputación y sin considerar la exéresis de falanges), de enfermedad renal crónica (ERC) y la Escala de Wagner para pie diabetico. Cabe destacar que esta escala es empleada para la cuantificación de lesiones tróficas en el pie diabético, clasificadas en seis grados según Wagner-Merrit ${ }^{[14]}$. Se consideró los valores registrados en la ficha de los tres meses previos al inicio del seguimiento.

\section{Análisis estadístico}

Se realizó un análisis descriptivo mostrando la media y desviación estándar de las variables numéricas; y los porcentajes y las frecuencias de las variables categóricas, incluyendo las variables principales como fallecimiento durante el estudio, comorbilidades y variables sociodemográficas. Luego, se hizo un análisis bivariado en base a supervivencia al final del estudio donde se analizaron todas las covariables en base a la variable principal del estudio. Las variables numéricas fueron analizadas mediante la prueba t de Student comparando el promedio de ellas en ambos grupos, previa corroboración de la distribución normal de éstas.

Para las variables categóricas, no se pudo utilizar la técnica de Chi cuadrado pues no se cumplía con el supuesto estadístico de tamaño muestral mínimo por cada celda, por lo que se acudió a la prueba exacta de Fisher. Se consideró un valor $p<0,05$ como estadísticamente significativo.

\section{Aspectos éticos}

El presente es un análisis secundario de base de datos, por lo que no se tuvo acceso a la información personal de los participantes. Esta investigación cuenta con aprobación por el Comité Institucional de Ética en Investigación de la Universidad Científica del Sur, con Código de Registro: 337-2018-PRE15.

\section{RESULTADOS}

La edad promedio de los participantes fue de 79,3 $\pm 7,2$ años, hubo una predominancia del sexo masculino con $58,4 \%$ sobre el femenino con $41,6 \%$. La media del tiempo de enfermedad fue de 24,0 $\pm 7,1$ años; asimismo, encontramos que 52 participantes $(58,4 \%)$ tenían antecedentes de albuminuria y $7,9 \%$ de la población falleció durante el estudio (Tabla 1).

Existen diferencias estadísticamente significativas entre albuminuria y fallecimiento, evidenciándose que el $100 \%$ de pacientes fallecidos durante el estudio presentaban albuminuria. Adicionalmente, se encontró que el promedio de edad y de tiempo en años con diagnóstico de DM2 era significativamente mayor en el grupo de pacientes fallecidos. Además, se evidenció en los pacientes que fallecieron solamente se encontraron personas con estado civil viudo o soltero (Tabla 2).

Por otro lado, los pacientes con un control no adecuado de la DM2 tenían una frecuencia de fallecimiento mayor en comparación a los que tenían un control adecuado. Asimismo, se encontró que los pacientes que tenían un nivel educativo 
Tabla 1. Características sociodemográficas y clínicas de los participantes del estudio ( $n=89$ ).

\begin{tabular}{|c|c|}
\hline Variables & $n(\%)$ \\
\hline Edad (años) $)^{* *}$ & $79,3 \pm 7,2$ \\
\hline Tiempo de diagnóstico (años) ${ }^{(*)}$ & $24,0 \pm 7,1$ \\
\hline \multicolumn{2}{|l|}{ Sexo } \\
\hline Femenino & $37(41,6)$ \\
\hline Masculino & $52(58,4)$ \\
\hline \multicolumn{2}{|l|}{ Estado Civil } \\
\hline Soltero/a & $9(10,2)$ \\
\hline Casado/a & $36(40,9)$ \\
\hline Viudo/a & $30(34,1)$ \\
\hline Divorciado/a & $13(14,8)$ \\
\hline \multicolumn{2}{|l|}{ Control de diabetes } \\
\hline Adecuado & $74(83,1)$ \\
\hline No adecuado & $15(16,9)$ \\
\hline \multicolumn{2}{|l|}{ Grado de Instrucción } \\
\hline Analfabeto/Incompleto & $21(23,6)$ \\
\hline Secundaria Completa & $39(43,8)$ \\
\hline Educación técnica & $28(31,5)$ \\
\hline Superior & $1(1,1)$ \\
\hline \multicolumn{2}{|l|}{ Escala de Wagner } \\
\hline Estadío I & $32(36,0)$ \\
\hline Estadío II & $35(39,3)$ \\
\hline Estadío III & $11(12,4)$ \\
\hline Estadío IV & $11(12,4)$ \\
\hline \multicolumn{2}{|l|}{ Enfermedad Renal Crónica } \\
\hline No & $26(29,9)$ \\
\hline Sí & $61(70,1)$ \\
\hline \multicolumn{2}{|l|}{ Albuminuria } \\
\hline No & $37(41,6)$ \\
\hline Sí & $52(58,4)$ \\
\hline \multicolumn{2}{|l|}{ Fallecimiento durante el estudio } \\
\hline No & $82(92,1)$ \\
\hline Sí & $7(7,9)$ \\
\hline \multicolumn{2}{|l|}{ Amputación de miembros inferiores } \\
\hline No & $62(69,7)$ \\
\hline Sí & $27(30,3)$ \\
\hline
\end{tabular}

${ }^{*}$ Promedio \pm desviación estándar

promedio a bajo, secundaria completa o incompleta/ analfabeto, tenían cifras elevadas de fallecimiento $(28,6 \%$ y $71,4 \%$, respectivamente).
Al igual que en el caso de la albuminuria, todos los pacientes que fallecieron durante el seguimiento contaban con el antecedente de amputación de miembros inferiores debido a complicación del pie diabético. Es así, que respecto a la escala de Wagner se observa que, a peor estadiaje, la mortalidad se duplicaba.

\section{DISCUSIÓN}

En esta investigación exploramos la utilidad del antecedente de albuminuria como un factor independiente de riesgo de mortalidad, encontrando una mortalidad muy elevada durante los 5 años de seguimiento, donde el $100 \%$ de pacientes fallecidos tuvo antecedente de albuminuria. Estos hallazgos son coherentes con un estudio norteamericano longitudinal llevado a cabo por Berhane et al. (2011) ${ }^{[15]}$ en pacientes diabéticos durante 23 años, donde señalan que el valor cuantitativo de la albuminuria es significativo como pronóstico para el desarrollo de enfermedad renal crónica, lo que incrementa el riesgo de muerte de hasta 1,37 veces.

En un metaanálisis realizado por Matsushita et al. (2017) ${ }^{[16]}$ en 817084 pacientes, provenientes de 21 cohortes, principalmente diabéticos, sin antecedente de enfermedad arterial periférica, con una media de 54 años y seguidos durante 7,4 años, determinaron una fuerte asociación entre albuminuria con la incidencia de enfermedad arterial periférica. Es así que, con valores de albuminuria de $300 \mathrm{mg}$, el riesgo de enfermedad vascular aumentaba 2,28 veces y la amputación de miembros inferiores era de 3,68 veces más. Se observó que, de manera similar a nuestros resultados, un estudio en pacientes con pie diabético publicado por Akther et al. (2011) ${ }^{[17]}$ encontró que el estadio de Wagner de mayor prevalencia fue el II, apreciándose que la supervivencia disminuye a mayor estadiaje, sobre todo en presencia de gangrena local o IV grado (8,5\%).

Se evidenció que el total de pacientes fallecidos habían requerido ser intervenidos con amputación de miembros inferiores, lo que concuerda con el alto riesgo de muerte en el postoperatorio como señalan diversos autores ${ }^{[18,19]}$, quienes sostienen una mortalidad de aproximadamente $52-80 \%$ en los primeros 5 años, encontrándose que la mortalidad aumentaba $7 \%$ por año tras amputación y la presencia de enfermedad cardiovascular.

Dentro de nuestro estudio se halló que dentro del grupo de pacientes fallecidos la mayoría se encontraba en situación de vulnerabilidad social por estado civil o grado de instrucción, con una proporción mayor de fallecidos entre aquellos adultos mayores solteros, viudos o con instrucción deficiente, por lo que sostenemos que en el contexto del adulto mayor con pie diabético el soporte social juega un rol importante el cual favorece la adherencia, el autocuidado, la evolución clínica y por ende disminuye la mortalidad ${ }^{[20,21]}$.

En relación al tiempo de enfermedad, un estudio encontró que el tiempo de diagnóstico no era diferente en pacientes con y sin pie diabético, por lo cual esta variable sería influenciada por otras 
Tabla 2. Análisis bivariado de factores asociados a Mortalidad en la población de estudio ( $n=89)$.

\begin{tabular}{|c|c|c|c|}
\hline Variables & $\begin{array}{l}\text { Sobrevivientes } \\
\quad(n=82)\end{array}$ & $\begin{array}{l}\text { Fallecidos } \\
\qquad(n=7)\end{array}$ & Valor $p$ \\
\hline Edad (años) * & $78,6 \pm 7,0$ & $87,1 \pm 4,4$ & $0,002^{+}$ \\
\hline Tiempo de diagnóstico (años) * & $23,4 \pm 7,0$ & $31,4 \pm 4,8$ & $0,004^{+}$ \\
\hline Sexo & & & $0,900 \ddagger$ \\
\hline Femenino & $34(41,5)$ & $3(42,9)$ & \\
\hline Masculino & $48(58,5)$ & $4(57,1)$ & \\
\hline Estado Civil & & & $0,020 \ddagger$ \\
\hline Soltero/a & $7(8,6)$ & $2(28,6)$ & \\
\hline Casado/a & $36(44,4)$ & $0(0,0)$ & \\
\hline Viudo/a & $25(30,9)$ & $5(71,4)$ & \\
\hline Divorciado/a & $13(16,1)$ & $0(0,0)$ & \\
\hline Control de diabetes & & & $<0,001 \ddagger$ \\
\hline Adecuado & $73(89,0)$ & $1(14,3)$ & \\
\hline No adecuado & $9(11,0)$ & $6(85,7)$ & \\
\hline Grado de Instrucción & & & $0,020 \ddagger$ \\
\hline Analfabeto/Incompleto & $16(19,5)$ & $5(71,4)$ & \\
\hline Secundaria Completa & $37(45,1)$ & $2(28,6)$ & \\
\hline Educación técnica & $28(34,1)$ & $0(0,0)$ & \\
\hline Superior & $1(1,2)$ & $0(0,0)$ & \\
\hline Escala de Wagner & & & $0,001 \ddagger$ \\
\hline Estadío I & $32(39,0)$ & $0(0,0)$ & \\
\hline Estadío II & $34(41,5)$ & $1(14,3)$ & \\
\hline Estadío III & $9(11,0)$ & $2(28,6)$ & \\
\hline Estadío IV & $7(8,5)$ & $4(57,1)$ & \\
\hline Enfermedad renal crónica & & & $0,300 \ddagger$ \\
\hline No & $25(31,2)$ & $1(14,3)$ & \\
\hline Sí & $55(68,8)$ & $6(85,7)$ & \\
\hline Albuminuria & & & $0,020 \ddagger$ \\
\hline No & $37(45,1)$ & $0(0,0)$ & \\
\hline Sí & $45(54,9)$ & $7(100,0)$ & \\
\hline Amputación de miembros inferiores & & & $0,001 \ddagger$ \\
\hline No & $62(75,6)$ & $0(0,0)$ & \\
\hline Sí & $20(24,4)$ & $7(100,0)$ & \\
\hline
\end{tabular}

*Promedio \pm desviación estándar

+T de Student

¥ Prueba exacta de Fisher

comorbilidades, especialmente la enfermedad cardiovascular y el control glicémico ${ }^{[22,23]}$. Se sabe que éste es un factor asociado a mejor pronóstico de evolución de úlceras por pie diabético, prevención de amputaciones, y mortalidad por causa cardiovascular en estos pacientes. Sin embargo, la mayoría de estudios sobre control glicémico son realizados en poblaciones más jóvenes, lo que no refleja, en su totalidad, las complejidades del adulto mayor tales como la fragilidad y otras comorbilidades asociadas, como la ERC ${ }^{[24]}$.

Adicionalmente, los estudios ACCORD, VADT y ADVANCE han demostrado que un control glicémico estricto en el adulto mayor no reduce la morbimortalidad cardiovascular sino, por el contrario, incrementa la frecuencia de aparición de 
otras complicaciones como la hipoglicemia ${ }^{[25-27]}$. Por tanto, los objetivos glicémicos deben individualizarse tomando en consideración el estado funcional de cada paciente ${ }^{[28]}$. En el contexto de nuestro estudio, es posible que el punto de corte empleado como un control adecuado de diabetes ( $\mathrm{HbA} 1 \mathrm{c}<7 \%)$ no sea el más adecuado para predicción de mortalidad en la población de adultos mayores con fragilidad en general. Por ello, es que la Asociación Americana de Diabetes (ADA 2019), el Colegio Americano de Médicos (ACP) y la Sociedad Americana de Geriatría ${ }^{[29]}$ plantean el uso de valores más conservadores como control óptimo, sugiriendo considerar puntos de corte de $\mathrm{HbA} 1 \mathrm{c}$ entre 7,5-8\% para el adulto mayor frágil, tomando en consideración el uso de polifarmacia, serias comorbilidades y la expectativa de vida de manera individual.

En las limitaciones del estudio se reconoce que nuestro tamaño muestral es reducido; sin embargo, la población de adultos mayores que se puede atender de manera ambulatoria es limitada. Asimismo, nuestra población pertenece a un hospital militar; por tanto, nuestras conclusiones podrían no ser extrapolables al total de casos de pie diabético en nuestro medio, especialmente en aquellos que no pueden acceder a un tratamiento y seguimiento continuo. Además, debemos mencionar que no se contaba con el valor de depuración de creatinina en el registro de historia clínica, valorando la función renal únicamente mediante la presencia de microalbuminuria.

Finalmente, en el análisis estadístico evidenciamos una baja frecuencia de mortalidad encontrándose, como se ha mencionado previamente, que todos los pacientes que fallecieron durante el seguimiento presentaron microalbuminuria, lo que impidió la realización de estadística multivariada como un análisis de regresión para determinar el riesgo de mortalidad en pacientes con albuminuria.

Se sugiere que en unidades especializadas en el manejo de este tipo de pacientes complejos, se realice una evaluación integral de manera rutinaria identificando oportunidades terapéuticas clave desde la cuantificación de la albuminuria y función renal para el correcto estadiaje de enfermedad renal crónica hasta el bienestar psicosocial del paciente. En adición, nuevos estudios que incluyan un mayor número de casos representativos de las distintas realidades de acceso a servicios de salud en nuestro país brindarían la posibilidad de realizar un análisis multivariado, con la generación de evidencia extrapolable en nuestro medio.

Se concluye que, en el contexto de nuestros pacientes, la presencia de albuminuria estuvo relacionada con el fallecimiento en adultos mayores con pie diabético; sin embargo, no se puede suponer que esto se deba exclusivamente al grado de afectación renal, sino a una estrecha relación con las múltiples comorbilidades que van en detrimento de la calidad de vida, destacando la presencia de amputación y la edad avanzada.

Contribuciones de autoría: los autores indican haber participado durante la formulación del problema, la recolección de los datos, el análisis de los mismos, la redacción de este artículo y la aprobación de la versión final.
Potenciales conflictos de interés: los participantes niegan conflictos de interés

Fuentes de financiamiento: autofinanciado.

\section{ORCID:}

Fernando M. Runzer-Colmenares: https://orcid.org/0000-00034045-0260

José F. Parodi: https://orcid.org/0000-0002-0336-0584

\section{REFERENCIAS BIBLIOGRÁFICAS}

1. Villena J. Diabetes Mellitus in Peru. Annals of Global Health. 2015;81(6):765-775. doi: 10.1016/j.aogh.2015.12.018.

2. Ramos W, López T, Revilla L, More L, Huamaní M, Pozo M. Resultados de la vigilancia epidemiológica de diabetes mellitus en hospitales notificantes del Perú, 2012. Rev Perú Med Exp Salud Pública. 2014;31(1):9-15. doi: 10.17843/rpmesp.2014.311.2.

3. Guerrero-Godínez JM, Barragán-Vigil AM, Navarro-Macías CL, Murillo-Bonilla LM, Uribe-González RP, Sánchez-cruz MA. Diabetes Mellitus en el Adulto Mayor. Rev Med Clin. 2017;1(2):81-94. doi: 10.5281/zenodo.1186879.

4. Neyra-Arisméndiz L, Solís-Villanueva J, Castillo-Sayán Ó, GarcíaRamos F. Pie Diabético. Rev Soc Peru Med Interna. 2012;25(2):76-88.

5. Majul E, Camps D. Microalbuminuria en diabetes tipo 2: signo de riesgo y oportunidad terapéutica. Archivos de Medicina [Internet]. 2007 [citado 24 mayo 2020];3(1). Disponible en: http://imed.pub/ ojs2/index.php/archmed/article/view/24.

6. Guerrero-Romero F, Rodríguez-Moran M. Relationship of Microalbuminuria with the Diabetic Foot Ulcers in Type II Diabetes. Journal of Diabetes and Its Complications. 1998;12:193-196. doi: 10.1016/s1056-8727(97)00112-8.

7. Akha O, Kashi Z, Makhlough A. Correlation Between Amputation of Diabetic Foot and Nephropathy. Iranian Journal of Kidney Diseases. 2010;4(1): 27-31.

8. Ramirez Chávez B. Factores de Riesgo para Amputación en Pacientes Geriátricos con Diagnóstico de Pie Diabético Centro Médico Naval 2010-2015. [Tesis para título de médico cirujano]. Lima, Perú: Universidad San Martin de Porres; 2017.

9. Runzer Colmenares FM. Fragilidad en Adultos Mayores y su Asociación con Dependencia funcional. [Tesis para título de especialista en Geriatría]. Lima, Perú: Universidad San Martin de Porres; 2012.

10. Matsushita K, van der Velde M, Astor BC, Woodward M, Levey AS de Jong $\mathrm{PE}$, et al. Association of estimated glomerular filtration rate and albuminuria with all-cause and cardiovascular mortality: a collaborative meta-analysis of general population cohorts. Lancet. 2010;375(9731):2073-81. doi: 10.1016/S0140-6736(10)60674-5

11. Dean AG, Sullivan KM, Soe MM. OpenEpi: Open Source Epidemiologic Statistics for Public Health [Internet]. 2013 [citado 30 octubre 2019]. Disponible en: http://www.OpenEpi.com

12. Tagle R, González F, Acevedo M. Microalbuminuria y excreción urinaria de albúmina en la práctica clínica. Rev Med Chile. 2012;140(6):797-805. doi: 10.4067/S0034-98872012000600016.

13. American Diabetes Association. Glycemic targets: Standards of Medical Care in Diabetes-2020. Diabetes Care. 2020; 43(Suppl 1):S66-S76. doi: $10.2337 / \mathrm{dc} 20-$ S006. 
14. Del Castillo Tirado RA, Fernández López JA, Del Castillo Tirado FJ. Guía de Práctica Clínica en el Pie Diabético. Archivos de Medicina. 2014;10(2):1. doi: 10.3823/1211.

15. Berhane AM, Weil EJ, Knowler WC, Nelson RG, Hanson RL. Albuminuria and Estimated Glomerular Filtration Rate as Predictors of Diabetic End-Stage Renal Disease and Death. Clin J Am Soc Nephrol. 2011;6(10):2444-2451. doi: 10.2215/CJN.00580111.

16. Matsushita K, Ballew SH, Coresh J, Arima H, Ärnlöv J, Cirillo M, et al. Measures of chronic kidney disease and risk of incident peripheral artery disease: a collaborative meta-analysis of individual participant data. Lancet Diabetes Endocrinol. 2017;5(9):718-28. doi: 10.1016/S2213-8587(17)30183-3

17. Akther JM, Khan IA, Shahpurkar VV, Khanam N, Syed ZQ. Evaluation of the diabetic foot according to Wagner's classification in a rural teaching hospital. Br J Diabetes Vasc Dis. 2011;11(2):74-79. doi: $10.1177 / 1474651411406372$

18. Thorud JC, Seidel JL. A Closer Look at Mortality after Lower Extremity Amputation. Podiatry Today. 2018;31(4):12-16.

19. Kristensen MT, Holm G, Kirketerp-Møller K, Krasheninnikoff M, Gebuhr P. Very low survival rates after non-traumatic lower limb amputation in a consecutive series: what to do? Interact Cardiovasc Thorac Surg. 2012;14(5):543-547. doi: 10.1093/icvts/ivr075.

20. Zhang X, Norris SL, Gregg EW, Beckles G. Social Support and Mortality Among Older Persons With Diabetes. Diabetes Educ. 2007;33(2):273-81. doi: 10.1177/0145721707299265.

21. Strom JL, Egede LE. The Impact of Social Suppot on Outcomes in Adult Patients with Type 2 Diabetes: A Systematic Review. Curr Diab Rep. 2012;12(6):769-781. doi: 10.1007/s11892-012-0317-0.

22. Sellman A, Katzman P, Andreasson S, Löndahl M. Presence of chronic diabetic foot ulcers is associated with more frequent and more advanced retinopathy. Diabet Med. 2018;35(10):1364-70. doi: $10.1111 /$ dme.13682
23. Steinarsson AO, Rawshani A, Gudbjörnsdottir S, Franzén S, Svensson A-M, Sattar N. Short-term progression of cardiometabolic risk factors in relation to age at type 2 diabetes diagnosis: a longitudinal observational study of 100,606 individuals from the Swedish National Diabetes Register. Diabetologia. 2018;61(3):599-606. doi: 10.1007/s00125-017-4532-8.

24. García-Esquinas E, Graciani A, Guallar-Castillón P, López-García $E$, Rodríguez-Mañas L, Rodríguez-Artalejo F. Diabetes and risk of frailty and its potential mechanisms: a prospective cohort study of older adults. J Am Med Dir Assoc. 2015;16(9):748-54. doi: 10.1016/j.jamda.2015.04.008.

25. Action to Control Cardiovascular Risk in Diabetes Study Group, Gerstein HC, Miller ME, Byington RP, Goff DC, Bigger JT, et al. Effects of intensive glucose lowering in type 2 diabetes. $N$ Engl J Med. 2008;358(24):2545-59. doi: 10.1056/NEJMoa0802743.

26. ADVANCE Collaborative Group, Patel A, MacMahon $S$, Chalmers $J$, Neal B, Billot L, et al. Intensive blood glucose control and vascular outcomes in patients with type 2 diabetes. N Engl J Med. 2008;358(24):2560-72. doi: 10.1056/NEJMoa0802987.

27. Duckworth W, Abraira C, Moritz T, Reda D, Emanuele N, Reaven PD, et al. Glucose Control and Vascular Complications in Veterans with Type 2 Diabetes. New England Journal of Medicine. Massachusetts Medical Society. 2009;360(2):129-39. doi: 10.1056/ NEJMoa0808431.

28. Gómez Huelgas R, Díez-Espino J, Formiga F et al. Tratamiento de la diabetes tipo 2 en el paciente anciano. Med Clin (Barc). 2013;140(3):89-90. doi: 10.1016/j.medcli.2012.10.003.

29. Longo M, Bellastella G, Maiorino MI, Meier JJ, Esposito K, Giugliano D. Diabetes and Aging: From Treatment Goals to Pharmacologic Therapy. Front Endocrinol (Lausanne). 2019;10. doi: 10.3389/ fendo.2019.00045 